\title{
Commentary on: Solberg and Jensenius (2016) Investigation of 'intersubjectively embodied experience' in a controlled electronic dance music setting
}

\author{
ALINKA E. GREASLEY[1] \\ School of Music, University of Leeds
}

\begin{abstract}
The motion capture study conducted by Solberg and Jensenius 2016 explores how people's movement on a dance floor is shaped by structural features of electronic dance music (EDM), with a particular focus on the breakdown and drop sections of tracks, the former being characterised by a reduction in musical texture and removal of the rhythmic bassline, and the latter when the rhythmic bassline re-enters and a thicker texture is re-established. The research also explored links between how the participants moved to the dance tracks and their experience of pleasure, and examined the extent to which dancing to EDM can be described as intersubjective. Results showed that the quantity of the dancers' movement reduced during the breakdown, and increased at the drop, as predicted. Ratings of enjoyment of the tracks, and qualitative reflections from the participants provided supporting evidence that the desire to dance increased at the moment of the drop, and that this was influenced by the movement of other people in the setting. In this commentary, I provide an overview of the authors' main arguments and critically evaluate the method and findings with suggestions for future studies.
\end{abstract}

Submitted 2016 June 6; accepted 2016 August 21.

KEYWORDS: electronic dance music, pleasure, movement, dancing, motion capture

\section{CONTEXT}

SOLBERG and Jensenius (2016) note that the majority of previous research on the shared experience of dancing in club settings has focused on sociological and cultural factors such as identity, gender and sexuality (e.g. Thornton, 1995; St John, 2004), and one of the latest texts in the field of EDM, 'DJ Culture: In the Mix' edited by Attias, Gavanas and Rietveld (2013) attests to the predominance of approaches focusing on broader cultural factors. The authors draw out a number of implications from research in the field of music psychology to contextualize their study. First, there is a growing body of literature on how people synchronize movements to music (e.g. Gritten \& King, 2011; Repp \& Su, 2013) and how this may be affected by emotional responses to music (e.g. Burger, Saarikallio, Luck, Thompson \& Toiviainen, 2013; Godøy \& Leman, 2010; Leman, 2007) but little is known about the ways in which people move to EDM. Second, peak pleasure sensations are experienced when musical expectations are fulfilled or violated, relating to sudden changes in the dynamics, texture and tempo of music (e.g. Huron, 2006, Gebauer, Kringelbach, Vuust, Cohen \& Stewart, 2012), but the role of anticipation and expectation in pleasurable responses to EDM has not received sufficient attention. Third, research has demonstrated links between enjoyment of music and movement (e.g. Janata, Tomic \& Haberman, 2012; Witek, 2013) and the authors propose that dancing to EDM represents a 'dynamic understanding of pleasure' whereby the experience of pleasure and the desire to move co-occur and reinforce each other. Fourth, research has shown that people are able to synchronize their movement more accurately in group settings (e.g. Godøy \& Leman, 2010) and that dancing can promote social bonding (e.g. Becker, 2004), leading Solberg and Jensenius to describe dancing to EDM in a club setting as an intersubjectively embodied experience.

Solberg and Jensenius (2016) devised a motion capture study to explore some of these issues. Specifically, they wanted to test the following predictions: 1 ) that participants' movements would differ in response to different passages of the break routine of tracks (breakdown, build-up, drop), with increased movement after the drop; 2) that there would be a relationship between pleasurable experiences and structural features of the music; and 3) that there would be evidence of intersubjective embodied understanding of the musical features of EDM. In order to test these predictions, Solberg (first author) pre-recorded a 15 minute 
mix that contained four house tracks: two tracks with breakdowns and two tracks without breakdowns. Participants were fitted with two reflective markers each, positioned on their head and attached to their dominant wrist, and were asked to dance to the music as they normally would in a club setting. Their dancing was tracked and recorded using infrared, marker-based motion capture. After this, participants were asked to complete a survey about their music listening and dancing habits, and asked to rate preference for the four tracks used in the study. Quantity of movement was then mapped onto the amplitude and spectrogram of the music. The sixteen participants were aged 22-54 years old, which reflects the general age range of clubbers.

\section{CONSTRUCTING A NIGHTCLUB SETTING IN A LABORATORY}

The authors transformed the motion capture laboratory into as realistic a nightclub setting as possible. This included a dance floor marked out by a piece of black carpet, appropriate lighting, and the pre-recorded mix played loudly through a 42-channel surround sound system. The colored lighting, programmed to synchronize to the beat of the music, is typical of most club settings, as is having the main lights turned off, which serves to reduce visual perception and heighten auditory sensation (cf. Fikentscher, 2000). Having the lights turned off may also have helped participants feel less embarrassed when dancing in close proximity with strangers. One missing element in pursuit of an ecologically valid nightclub setting was the DJ. Historically, DJs have tended to occupy a booth in the corner of a nightclub and are not always visible to clubbers on the dancefloor; it could therefore be argued that dancing to a mix without seeing a DJ is not an atypical experience. However, DJs have become more of a focal point than they used to be, with booths being raised up so that the DJ is visible, and the DJ taking a performative role (Rietveld, 2013). DJs respond to people's movement on the dancefloor in real-time and alter their choice of tracks to encourage dancing (Broughton \& Brewster, 2002; Greasley \& Prior, 2013). In addition to their musical choices and mixing skills, the DJs move themselves and 'hype' the crowd (see for example, Martin Garrix's Forbidden Voices video)[2]. A further consideration, which the authors acknowledge, is that a proportion of people in nightclubs are likely to have been drinking or taking recreational drugs. In real settings, this is likely to have an effect on the extent and range of movement on the dancefloor and also their interaction with others.

\section{STRUCTURAL ANALYSIS AND QUANTITY OF MOTION}

Analyses had to be conducted for all participants together, as it was not possible to track the movement of individual participants due to marker occlusion (participants were constantly moving, raising their arms, and dancing close to each other). Results show clear patterns between key structural features of the music, selfreported pleasure and quantity of motion, with marked differences between the control tracks (Joyride, Unlock Down) and the tracks with break routines (Ladykiller, Icarus).

Various graphs are presented which compare frequency $(\mathrm{Hz})$ and amplitude of the musical signal with average quantity of motion over time. These show that when participants were dancing to the control tracks, their movement did not vary greatly, whereas for the two tracks with break routines, there was a marked decrease in movement at the breakdown, and a significant increase in movement at the drop. A more pronounced effect was found for the track Icarus, which is likely due to the structural properties of the breakdown. Ladykiller thins in texture at the breakdown but a steady pulse remains, whereas the texture is stripped back in Icarus, and there is a lengthier build up to the drop. The average quantity of motion for Icarus before the breakdown was lower than the average quantity of motion after the breakdown, suggesting that the break routine energized the participants. Icarus was rated as the most pleasurable out of the four tracks. Participants reported that their desire to dance was due to a strong, regular beat, and that the intense desire to dance experienced was related to changes in dynamics and/or structural properties of the music.

These findings raise a number of questions and potential avenues for further research. The prerecorded mix was purposefully designed so that the first and third tracks had little structural development, and the second and fourth had break routines. It would be interesting to explore different ordering of tracks. If a track with a break routine is played after three structurally basic tracks (what are referred to by DJs as 'filler' tracks), is there greater movement at the drop? If dancers had experienced no 'build up' or 'drop' within the last few tracks, one might expect that their movement would be more energetic. It would also be interesting to explore the DJ technique known as 'double drop', which is when the DJ mixes two tracks together such that the drop in each track occurs at the same time. Does this result in heightened pleasurable experiences? Is the quantity of motion proportionally greater when two tunes 'drop' together? 
Solberg and Jensenius used house music, and whilst they controlled for preference to some extent by asking participants to listen to the tracks afterwards and rate how pleasurable they found them, it is likely that people who prefer faster-paced forms of dance music (e.g. drum 'n' bass) will be less motivated to move to house music (with its slower beats per minute) than those whose preferred style is house. Future research should explore movement to the break routines of a broader range of styles such as techno, trance and drum ' $n$ ' bass. Strength of preference for style should be controlled for in design. It would also be of value to explore similarities and differences at a more fine-grained level since there are considerable differences in the sonic features of sub-styles of genres. For example, within drum ' $n$ ' bass there are many sub-styles such as tech, neurofunk, liquid, jungle and ambient drum ' $n$ ' bass. Do people move in jerkier ways to the 'amen breaks' of jungle and move with fluid motions when dancing to liquid drum ' $n$ ' bass?

Another focus could be the special effects (e.g. reverb, echo, flange, looping) used by the DJ in the mix, and how these influence movement on the dancefloor. DJs report using special effects at key structural points of tracks to sustain momentum and build tension (Greasley \& Prior, 2013). Research could also focus on the use of 'looping' to prolong the 'build up' before the 'drop' which heightens anticipation, and tension/release. Another technique that appears to have a pronounced effect on people's movement is the 'tease' which is when a DJ drops a (typically well-known) track into an existing tune to energize the crowd. It would be interesting to collect data on changes in movement when 'teases' are introduced into the mix.

\section{THE CONTRIBUTION OF OTHERS TO THE DANCING EXPERIENCE}

Participants in Solberg and Jensenius' study reported that the setting was more realistic due to the presence of others, and that other people's movement had motivated their own dancing. This provides further support for an intersubjective embodied understanding of the musical features of EDM. To build on this, future work could include a DJ performing in real-time in the experimental setting. The DJ's body movement, and also his/her interaction with the crowd (e.g. eye contact, gestures), could be captured to investigate the DJ's role in promoting movement on the dancefloor. Recently, 7UP sponsored the design and implementation of a 'Concert for the Deaf'[3] in which a variety of auditory, visual and vibrotactile perception enhancers were used to create an immersive nightclub experience for deaf people. This included custom vibrating plates, speaker cone walls, and visualizations that were synchronized with the music [4]. In the main video, it is possible to see the role the DJ, Martin Garrix, plays in motivating the crowd when he is mixing, particularly at 'drop' sections when he jumps up and down in synchrony with the beat. A study could be developed in which a DJ performs tracks in a set order with a) little movement, other than the minimum needed to mix, b) typical movement, that they would normally engage in, and c) exaggerated movement to explore the effects on the extent of movement on the dancefloor. This type of design has been used to explore the role of body movement in expressive performance in Western Art music predominantly, but to my knowledge there are no studies on DJs. Solberg and Jensenius discuss the use of accelerometer-based motion capture for real settings, and this seems like a logical next step if we are to learn more about the relative contribution of features of music and the DJ in shaping movement on the dancefloor.

\section{CONCLUDING REMARKS}

There is a lack of psychological research into people's experiences and behavior in nightclub settings, and considerations of music-related movement, and notions of pleasure, affect, empathy and imitation in relation to EDM are welcome and timely. The study provides a good starting point for future research exploring how people's movement is related to structural features of various styles of EDM, and how the presence of others can influence this. Whilst specifics of the methodology need rethinking, as it would have been valuable to have data on each individual's movement to compare to the average across all participants, the graphical representation of differences in quantity of motion in the various sections of the break routine provided clear evidence of the link between features of the music and extent of movement, which was further supported by survey data. I have made various suggestions for future work. In particular, attention should be focused on the DJ and the role s/he plays in energizing dancers, in terms of creative choices (e.g. order of tracks, use of effects), their own body movement and degree of interaction with the crowd. 


\section{NOTES}

[1] Correspondence can be addressed to: Dr. Alinka Greasley, School of Music, University of Leeds, Leeds, LS2 9JT, UK, a.e.greasley@leeds.ac.uk.

[2] Martin Garrix, Forbidden Voices. https://www.youtube.com/watch?v=Zv1QV6lrc_Y

[3] 'Concert for the Deaf', sponsored by 7UP, https://www.youtube.com/watch?v=kvIpVSA81Bw

[4] 'Meet the Creators', https://www.youtube.com/watch?v=cUOpN0eIx-A

\section{REFERENCES}

Attias, B. A., Gavanas, A. \& Rietveld, H. C. (2013). (Eds). DJ Culture In the Mix: Power, Technology and Social Change in Electronic Dance Music. London: Bloomsbury Publishing.

Becker, J. (2004). Deep listeners: Music, emotion, and trancing. Bloomington: Indiana University Press.

Broughton, F., \& Brewster, B. (2002). How to DJ (Properly): The Art and Science of Playing Records. London: Bantam Press.

Burger, B., Saarikallio, S., Luck, G., Thompson, M. R., \& Toiviainen, P. (2013). Relationships between perceived emotions in music and music-induced movement. Music Perception, 30(5), 517-533.

https://doi.org/10.1525/mp.2013.30.5.517

Fikentscher, K. (2000). 'You better work! Underground dance music in New York City’ Hanover, NH, and London: Wesleyan University Press.

Gebauer, L., Kringelbach, M. L., Vuust, P., Cohen, A. J., \& Stewart, L. (2012). Ever-changing cycles of musical pleasure: The role of dopamine and anticipation. Psychomusicology: Music, Mind, and Brain, 22(2), 152-167. https://doi.org/10.1037/a0031126

Godøy, R. I \& Leman, M. (2010). Musical gestures: sound, movement and meaning. New York: Routledge.

Greasley A. E. \& Prior, H. M. (2013). Mixtapes and turntablism: DJs' perspectives on musical shape. Empirical Musicology Review, 8(1), 23-43. https://doi.org/10.18061/emr.v8i1.3921

Gritten A., \& King E. (2011). New Perspectives on Music and Gesture. Farnham: Ashgate.

Huron, D. (2006). Sweet anticipation: Music and the Psychology of Expectation. Cambridge, Massachusetts: MIT Press.

Janata, P., Tomic, S. T., \& Haberman, J. M. (2012). Sensorimotor coupling in music and the psychology of the groove. Journal of Experimental Psychology: General, 141, 54-75. https://doi.org/10.1037/a0024208

Leman, M. (2007). Embodied music cognition and mediation technology. Cambridge, Massachusetts: MIT Press.

Repp, B., \& Su, Y.-H. (2013). Sensorimotor synchronization: A review of recent research (2006-2012). Psychonomic Bulletin \& Review, 20(3), 403-452. https://doi.org/10.3758/s13423-012-0371-2

Rietveld, H. C. (2013). Journey to the light? Immersion, Spectacle and Mediation. In A. B. Attias, A. Gavanas, \& Rietveld, H. C. (2013). (Eds). DJ Culture In the Mix: Power, Technology and Social Change in Electronic Dance Music. (pp. 79-102). London: Bloomsbury Publishing. 
Solberg, R. T. \& Jensenius, A. R. (2016). Pleasurable and Intersubjectively Embodied Experiences of Electronic Dance Music. Empirical Musicology Review, 11(3-4), 301-318.

https://doi.org/10.18061/emr.v11i3-4.5023

St John, G. (2004). Rave culture and religion. London: Routledge.

Thornton, S. (1995). Club cultures: Music, media and subcultural capital. Cambridge: Polity Press.

Witek, M. A. G. (2013). '...and I feel good!’ The Relationship between body-movement, pleasure and groove in music. Unpublished doctoral dissertation, University of Oxford, UK. 